\title{
REVIEW
}

\section{Use of pharmacogenetics for predicting cancer prognosis and treatment exposure, response and toxicity}

\author{
Daniel L Hertz ${ }^{1,2}$ and Howard L McLeod ${ }^{1}$ \\ Cancer treatment is complicated because of a multitude of treatment options and little patient-specific information to help \\ clinicians choose appropriate therapy. There are two genomes relevant in cancer treatment: the tumor (somatic) and the patient \\ (germline). Together, these two genomes dictate treatment outcome through four processes: the somatic genome primarily \\ determines tumor prognosis and response while the germline genome modulates treatment exposure and toxicity. In this review, \\ we describe the influence of these genomes on treatment outcomes by highlighting examples of genetic variation that are \\ predictors of each of these four factors, prognosis, response, toxicity and exposure, and discuss the translation and clinical \\ implementation of each. Use of pre-treatment pharmacogenetic testing will someday enable clinicians to make individualized \\ therapy decisions about aggressiveness, drug selection and dose, improving treatment outcomes for cancer patients. \\ Journal of Human Genetics (2013) 58, 346-352; doi:10.1038/jhg.2013.42; published online 16 May 2013
}

Keywords: cancer; efficacy; personalized medicine; pharmacogenetics; prediction; prognosis; toxicity

\section{INTRODUCTION}

Oncologists have a variety of pharmacological treatment options for many tumor types. Large clinical trials are useful for determining which drug is superior across the patient population, but tell us little about which drug is best for an individual. Within a population of treated patients, there is substantial variability in response to a given drug. The unpredictability of response leads to administration of ineffective therapy for some patients, which is associated with the following types of costs. Cancer treatment can be quite expensive, and ineffective therapy is a waste of financial resources. Also, the cost to the patient's quality of life from treatment-related toxicity is acceptable only if the tumor is responding. Finally, there is an opportunity cost of treating a patient with an ineffective drug and allowing the tumor to continue to grow, making it more difficult to control with future lines of therapy. These costs make it critical that we develop strategies for selecting optimal treatments for individual patients.

When devising a treatment plan for a patient there are two relevant genomes: that of the patient's germline and the cancer cells. ${ }^{1}$ The tumor genome, referred to as the somatic genome, will mainly determine the features of the tumor such as its aggressiveness and sensitivity to treatment. The patient, or germline, genome primarily dictates how the body handles and reacts to the chosen treatment. In this review, we will discuss the influence of variability in the somatic and germline genome on prediction of prognosis, efficacy, exposure and toxicity (Figure 1, Table 1). For each topic we will use specific pharmacogenetic examples to highlight key aspects of discovery and translation.

\section{SOMATIC GENETICS}

A healthy cell turns cancerous by losing its ability to appropriately regulate its replication. This initial genetic aberration can be a simple change in DNA sequence or a change that impacts an entire gene or chromosomal region. This malignant cell is the seed from which a tumor grows, and the genome of this cell is the founder somatic genome. Through repeated cellular replication this somatic genome acquires additional abnormalities. ${ }^{2}$ Some of these acquired variants will further drive cancer progression, such as mutations in genes that are responsible for maintaining DNA replication fidelity or controlling metastatic spread. ${ }^{3}$ The initial and acquired aberrations determine the behavior of the primary tumor, its ability to metastasize and the treatment mechanisms that it will be sensitive to. Thus, prediction of tumor prognosis and response can be accomplished by understanding somatic genetics.

\section{Prognostic predictors}

Prediction of cancer outcome, or prognosis, is dependent on local tumor growth and distant tumor spread. It is not surprising that the hallmark prognostic factors used in cancer therapy are tumor size and

${ }^{1}$ UNC Institute for Pharmacogenomics and Individualized Therapy, University of North Carolina at Chapel Hill, Chapel Hill, NC, USA and ${ }^{2}$ Department of Clinical, Social and Administrative Sciences, College of Pharmacy, University of Michigan, Ann Arbor, MI, USA

Correspondence: Dr DL Hertz, Department of Clinical, Social and Administrative Sciences, College of Pharmacy, University of Michigan, 428 Church Street, Ann Arbor, MI 48109, USA.

E-mail: DLHertz@med.umich.edu

Received 10 April 2013; accepted 12 April 2013; published online 16 May 2013 


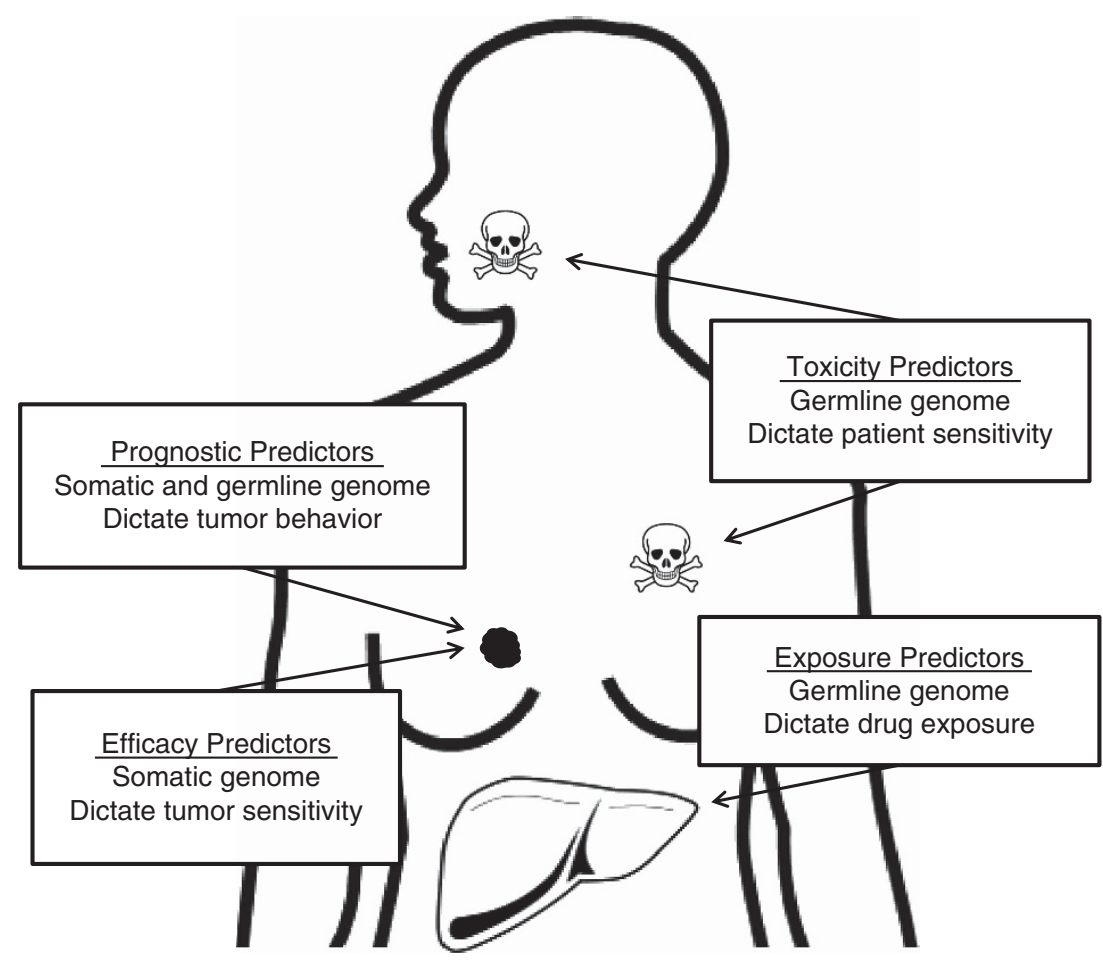

Figure 1 Pharmacogenetic predictors of cancer treatment outcome. Tumor prognosis is partially determined by variation in both the somatic and germline genome, which dictate tumor behavior independent of treatment. The somatic genome also dictates tumor sensitivity to treatment, enabling prediction of efficacy. The germline genome dictates drug pharmacokinetics (absorption, distribution, metabolism, excretion (ADME)) that can indirectly determine efficacy and toxicity, or the germline genome can directly influence patient sensitivity to toxicity.

Table 1 Prediction of cancer outcomes using germline and somatic genetic variation

\begin{tabular}{|c|c|c|c|c|}
\hline prediction & Genome & Genes & Treatment decision & Examples discussed \\
\hline Prognosis & Somatic or germline & $\begin{array}{l}\text { Oncogenes, tumor suppressors, DNA repair } \\
\text { mechanisms, metastasis genes }\end{array}$ & Aggressiveness & $\begin{array}{l}\text { Gene expression (breast cancer) BRCA2 (ovarian } \\
\text { cancer) }\end{array}$ \\
\hline Exposure & Germline & Enzymes, transporters & Dose & UGT1A1 (irinotecan and SN-38 exposure) \\
\hline Efficacy & Somatic & Tumor drivers, drug targets & Drug & BRAF V600E (vemurafenib) TSC1 (everolimus) \\
\hline Toxicity & Germline & $\begin{array}{l}\text { Drug targets, cellular response mechanisms, pro- } \\
\text { teins relevant to toxicity mechanism }\end{array}$ & Drug & $\begin{array}{l}\text { TUBB2A, FGD4, FANCD2 (paclitaxel-induced } \\
\text { peripheral neuropathy) }\end{array}$ \\
\hline
\end{tabular}

extent of metastasis at diagnosis. ${ }^{4}$ The aggressiveness with which a tumor will continue to spread is largely a consequence of somatic genetics. Understanding the genetic makeup of the tumor at diagnosis can tell us a lot about its aggressiveness and likely behavior in the future.

This information is clinically vital. Prognostic staging systems that utilize the above-mentioned clinical factors to dictate treatment aggressiveness exist for many tumor types. The addition of accurate genetic prognostic determinants to these staging systems will improve our ability to make appropriate decisions on treatment aggressiveness for individual patients. Patients with tumors with good prognosis can be treated less aggressively, or not at all, avoiding the secondary costs of unnecessary treatment. On the other hand, patients with tumors with poor prognosis can be treated aggressively, as the costs incurred are necessary to combat a dangerous disease. Somatic gene expression is one of the most widely used genetic tools for predicting cancer prognosis, particularly within breast cancer.
Breast cancer gene expression. Breast cancer treatment decisions are made by classification of tumors based on their expression of several receptors: estrogen, progesterone and HER2. An alternative system for classifying tumors into 'intrinsic subtypes' based on gene expression has been developed. ${ }^{5}$ The intrinsic subtypes, which are mostly concordant with receptor-based classification, have been demonstrated in independent patient populations to be predictive of disease recurrence, ${ }^{6}$ but gene expression analysis has not replaced receptor-based classification clinically. ${ }^{7}$

Two gene expression tools have been approved for use in the United States or Europe to help clinicians decide whether a patient should be offered chemotherapy after local therapy with surgery and/ or radiation. The first tool is OncotypeDx (Genomic Health, Inc., Redwood City, CA, USA), which was developed for hormonepositive, node-negative patients ${ }^{8}$ but may also be useful in low-risk, node-positive patients. ${ }^{9}$ The OncotypeDx system gives clinicians a recurrence score $(0-100)$, which is most often used clinically to 
identify patients with low-risk $(\mathrm{RS}<18)$ tumors, that can be treated with hormonal therapy alone. The ongoing TailoRx study is collecting data to help clinicians make treatment decisions for intermediate risk patients $(11 \leqslant \mathrm{RS} \leqslant 25) .{ }^{10}$ The Mammaprint (Agendia, NV, Amsterdam, The Netherlands) system was designed and validated for prognostic classification of premenopausal, node-negative patients $^{11,12}$ and has been applied to postmenopausal patients. ${ }^{13}$ Similar to OncotypeDx, clinical utility of Mammaprint continues to undergo investigation, notably in the prospective MINDACT study which is asking, among other things, what the best treatment approach is for the $\sim 1$ in 4 patients whose gene expression and clinical factors have discordant risk classification. ${ }^{14}$ Interestingly, despite the differences in the patients used for development and the genes included in each panel, the classification of patients using these three gene expression systems are pretty concordant. ${ }^{15}$

\section{Efficacy predictors}

It has long been recognized that some tumors are highly sensitive to specific treatments. However, because the cause of this sensitivity was not understood, efficacy prediction was unachievable. We now have the tools to identify the genetic aberrations present in a given tumor and it is apparent that in many cases tumors are highly sensitive to drugs that target the malignancy driver. The next revolution in cancer, which is currently underway, is to develop drugs that target the known oncogenic mutations and select treatments based on the genetic abnormalities present within a patient's tumor.

There are two principal pathways for this translational work. One is identifying the genetic biomarkers that confer tumor sensitivity to drugs currently in use and the other is to design drugs that specifically target oncogenic aberrations found in tumors. Either way, at the end of this process you have a drug that is highly effective in tumors that are known to possess a somatic biomarker that can be tested for before treatment initiation. Treatment individualization based on somatic biomarkers is being piloted at several cancer centers, ${ }^{16,17}$ but prospective validation of this approach is a major challenge. ${ }^{18}$ Eventually, this model could transform cancer treatment, enabling pre-treatment identification of patients who are likely to respond to a specific drug while sparing patients who are unlikely to respond.

Efficacy biomarker identification for existing drug. Everolimus, an inhibitor of the mammalian target of rapamycin, was first approved in 2009 for use in advanced kidney cancer. It has since been approved in limited treatment settings for a variety of tumor types, including pancreatic and breast cancer, and has been tested in a variety of other tumor types in clinical studies. Although some of these clinical studies failed to meet their efficacy end points, select patients experienced extremely impressive, durable responses.

Using samples collected from one of these studies, a group of investigators genotyped a metastatic bladder tumor from a patient who achieved a complete response that lasted $>2$ years. Many somatic mutations were present, including a deletion in the tuberus sclerosis 1 (TSC1) gene. Interestingly, TSC1 results in constitutive activation of mammalian target of rapamycin ${ }^{19}$ and everolimus is used to treated tuberous scleorisis, a disease characterized by mutation in the TSC1 gene. ${ }^{20,21}$ This intriguing finding led the investigators to screen more bladder tumors, and more TSC1 mutations were found. They then screened 13 patients from the original clinical study and found that the 6 patients with TSC1 mutations had superior time to response (4.1 vs 1.8 months; hazard ratio $(\mathrm{HR})=18.5(2.1-162), P=0.001)$ and remained on everolimus treatment longer $(7.7$ vs 2.0 months, $P=0.004){ }^{22}$ This is an exemplary case of biomarker discovery for a drug currently in use that awaits prospective validation and translation to clinical practice.

Drug developed to target a known oncogenic mutation. It has long been recognized that the RAS-RAF pathway is a major driver of many cancer types, including melanoma. ${ }^{23}$ This led to programs directed at finding chemical inhibitors of BRAF. One such program used a highthroughput screening approach and discovered vemurafenib, which was particularly selective for the most common activating mutation in BRAF (V600E). ${ }^{24}$ A phase I study was initiated in BRAF-mutated metastatic melanoma, which had extraordinarily promising results in this previously very difficult to treat tumor type. ${ }^{25}$ These results instigated an accelerated development program including a pivotal phase III trial that was closed early based on impressive interim results. ${ }^{26}$ Vemurafenib was approved soon thereafter with a validated companion diagnostic able to detect BRAF V600E-mutant melanomas. ${ }^{27}$

\section{GERMLINE GENETICS}

The patient's germline genome directs the processes functioning throughout the body by coding for the proteins involved. Thus, variability in the germline genome can have implications for any system operating within the patient. An inherited variant in a gene responsible for DNA replication fidelity or regulating cellular replication can predispose a patient to cancer, perhaps even a specific type. ${ }^{28}$ Alternatively, germline variation in genes encoding enzymes or transporters can have consequences for the patients exposure to the drugs administered ${ }^{29}$ or can influence a patient's sensitivity to the adverse effects of treatment. In this section, we describe the potential influence of germline variation on prediction of tumor prognosis, drug exposure or toxicity.

\section{Prognostic predictors}

Although most of the genetic determination of tumor prognosis takes place within the tumor genome, the patient genome can also have a role. It is widely accepted that many germline genetic loci contribute a small amount to the heritability of cancer, ${ }^{30,31}$ but it is unclear how many of these disease susceptibility loci have prognostic importance. The Cancer Genome Atlas (TCGA) is a large effort to categorize somatic mutation, expression, copy number and epigenetic modification within various tumor types. ${ }^{32}$ TCGA is also collecting germline DNA and limited outcome data, which enables researchers to look for associations between genetic variation and cancer prognosis.

TCGA has published their analyses of the first several tumor types including glioblastoma, ovarian, breast, lung and colorectal cancers. ${ }^{33-36}$ One interesting finding from the ovarian cancer analysis was a suggestion that patients carrying germline variants in the BRCA1 or 2 gene had superior survival compared with patients who did not carry a mutation in either gene. ${ }^{34}$ A more detailed analysis of this data confirmed specifically that patients who carried $B R C A 2$ mutations had superior overall and progression-free survival compared with other patients. ${ }^{37}$ A large pooled analysis from Bolton et al. ${ }^{38}$ reported that patients with BRCA2 mutation have the best 5 -year survival (52\%) followed by BRCA1 carriers (44\%) and then non-carriers $(36 \%)$.

There is also a possibility that germline genetics, through an influence on the tumor microenvironment, may influence tumor growth and metastasis. One intriguing line of research has identified several germline genes that may determine metastatic potential across cancer types: SIPA1, ${ }^{39-41} B R D 4^{42}$ and $R R P 1 B,{ }^{43,44}$ possibly through 
regulation of the Rap GTPase-activating protein SPA- $1 .{ }^{45}$ We are just now beginning to appreciate the influence of the tumor microenvironment on cancer progression, and we are a long way from fully understanding the role of host genetics on the behavior of the tumor in vivo.

\section{Exposure predictors}

The current chemotherapy treatment paradigm is to administer the dose that will maximize drug exposure, and hopefully treatment effectiveness, with an acceptable risk of toxicity. Unfortunately, most drugs have substantial interpatient pharmacokinetic $(\mathrm{PK})$ variability. ${ }^{46}$ For many drugs, the only factor that is incorporated into dosing decisions is patient size (body surface area (BSA), body weight, and so on) ${ }^{47}$ Body size explains some of the PK variability ${ }^{48}$ but the rest is a consequence of variability in absorption, metabolism, distribution and/or excretion (ADME) of the drug and metabolites.

For each drug there are several enzymes and transporters that carry out the ADME processes. The expression and activity of these enzymes and transporters are highly variable between patients, partially because of germline genetic variability. Germline variants in the coding region can change protein activity while variation outside of the coding region is more likely to influence protein expression.

Most pharmacogenetic association studies assess the relationship between variants and clinical outcomes; however, for variants in PK genes this indirect association is a consequence of a direct effect on drug exposure. Consequently, clinical implementation of these findings would typically be to modulate the patient's dosage in order to achieve optimal drug exposure. ${ }^{49}$

There are many examples of germline variants that may influence patient exposure to a cancer agent. ${ }^{50}$ In this section, we highlight one of the more well-established examples, the influence of germline polymorphisms on exposure to irinotecan and its active metabolite, SN-38.

UGT1A1 polymorphisms and irinotecan $(S N-38)$ exposure. Irinotecan is used in colorectal cancer and is associated with severe diarrhea and neutropenia. Both the efficacy and toxicities of irinotecan are attributable to $\mathrm{SN}-38$, which is formed when carboxyesterases bioactivate irinotecan. SN-38 is inactivated by glucuronidation via uridinediphosphate glucuronosyltransferase isoforms 1A1 (UGT1A1) and eliminated via biliary elimination, which is in part coordinated by the hepatic transporter OATP1B1 (SLCO1B1).

Transcription of UGT1A1 is variable among patients, partially due to germline variability in the promoter region. The number of thymine-adenine (TA) repeats in the TATA box is typically six but some patients have an additional dinucleotide repeat, known as $\mathrm{TA}_{7}$ or UGT1A1 ${ }^{\star} 28$, which diminishes glucuronidation activity. ${ }^{51}$ This led to investigations of whether patients carrying the ${ }^{\star} 28$ allele had differential exposure to SN-38. A small pilot study found, as anticipated, that the homozygous ${ }^{*} 28$ patient had the highest ratio of SN-38/SN-38 glucuronide. ${ }^{52}$ Since these initial studies many groups have reported analyses in large populations of irinotecan treated patients, most of which have detected an increase in active SN38 in patients who carry or are homozygous for the ${ }^{\star} 28$ allele. ${ }^{53-55}$ The influence on SN-38 exposure has also been extended to other UGT1A1 polymorphisms and haplotypes, and other UGT1A isoforms. ${ }^{56-59}$

As $\mathrm{SN}-38$ exposure determines efficacy and toxicity, low activity UGT1A1 polymorphisms are of potential clinical relevance. ${ }^{60,61} \mathrm{~A}$ relationship between UGT polymorphisms and irinotecan clinical outcome has been replicated in many patient cohorts, as reviewed by Di Paolo et al. ${ }^{62}$ Thus, translation of this pharmacogenetic association into clinical practice is underway. As explained previously, because the polymorphism is a predictor of drug exposure, the appropriate clinical solution would be to modify the dose for patients carrying low activity variants. But what is the ideal dose for patients of a given genotype?

Several groups have carried out genotype-guided early-phase clinical studies. Three studies in Japanese patients are difficult to interpret due to differences in toxicity definition and genotype grouping, and an upper limit of approved dosage $\left(150 \mathrm{mg} \mathrm{m}^{-2}\right)$ (Table 2 ). Two studies suggested that homozygous ${ }^{\star} 1$ patients could tolerate doses beyond $150 \mathrm{mg} \mathrm{m}^{-2}$ while ${ }^{\star} 28$ patients could not. ${ }^{63,64}$ However, a third study that excluded homozygous ${ }^{\star} 28$ patients reported that irinotecan could not be tolerated at $165 \mathrm{mg} \mathrm{m}^{-2}$, and the recommended dose for ${ }^{\star} 1$ carriers was set at $150 \mathrm{mg} \mathrm{m}^{-2}{ }^{65} \mathrm{On}$ the basis of the results of these dose escalation studies, Okuyama et al. conducted a phase II study in which ${ }^{\star} 28$ homozygous patients would be started at 100 instead of $150 \mathrm{mg} \mathrm{m}^{-2}$. Despite the dose decrease, the single UGT1A1*28 homozygous patient enrolled experienced grade 4 neutropenia. ${ }^{66}$

Two UGT1A1 genotype-guided dose escalation studies have been carried out in European patients. The recommended dose for ${ }^{\star} 1$ homozygous patients was $370-390 \mathrm{mg} \mathrm{m}^{-2}$ and for heterozygous patients $310-340 \mathrm{mg} \mathrm{m}^{-2}{ }^{67,68}$ Only Marcuello et al. enrolled ${ }^{\star} 28$ homozygous patients and recommended a dose of $130 \mathrm{mg} \mathrm{m}^{-2}$. In a genotype-guided phase II study, Freyer et al. ${ }^{69}$ were able to safely administer high-dose FOLFIRI, including irinotecan $260 \mathrm{mg} \mathrm{m}^{-2}$, to patients who carried UGT1A1*1 and had favorable thymidylate synthase genotypes.

These studies point the way toward irinotecan dose individualization based on factors other than BSA, which itself explains little PK variability. ${ }^{70}$ Beyond UGT1A1, there are many other enzymes and transporters involved in determining SN-38 exposure. Germline variation within and around these genes has also been evaluated for relationships with irinotecan $\mathrm{PK}$ and treatment outcome. ${ }^{71-74}$ In the future, pre-treatment pharmacogenetic analysis of multiple variants from these genes could enable clinicians to select appropriate doses for individual patients to target an optimal exposure of SN-38 that will achieve the greatest clinical benefit with acceptable risk of toxicity.

\section{Toxicity predictors}

Clinical factors, such as impaired liver function or drug interactions, influence a patient's likelihood of experiencing toxicity by affecting drug exposure, similar to that described in the previous section. Alternatively, at equivalent exposure some patients are highly sensitive to the adverse effects of certain treatments. In this section, we will discuss the influence of germline genetics on sensitivity to drug toxicity. As we know much less about pharmacology and pharmacodynamics than we do about PK, our understanding of the genetic predictors of toxicity lags far behind that of the genetic predictors of exposure previously discussed.

Sensitivity to toxicity could be a consequence of several biological processes. Cells that have high expression of a drug target or rely on that target for cellular homeostasis could be exquisitely sensitive to that drug. Alternatively, cells that are unable to mount the appropriate response to a stimulus, such as DNA damage, could be highly sensitive to chemotherapy that induces that stimulus. A third possibility, which exists not on the cellular but on the physiological scale, is the possibility that some patients have an underlying condition that mimics the toxicity, which is aggravated by the drug. 
Table 2 Genotype-guided irinotecan dose escalation studies

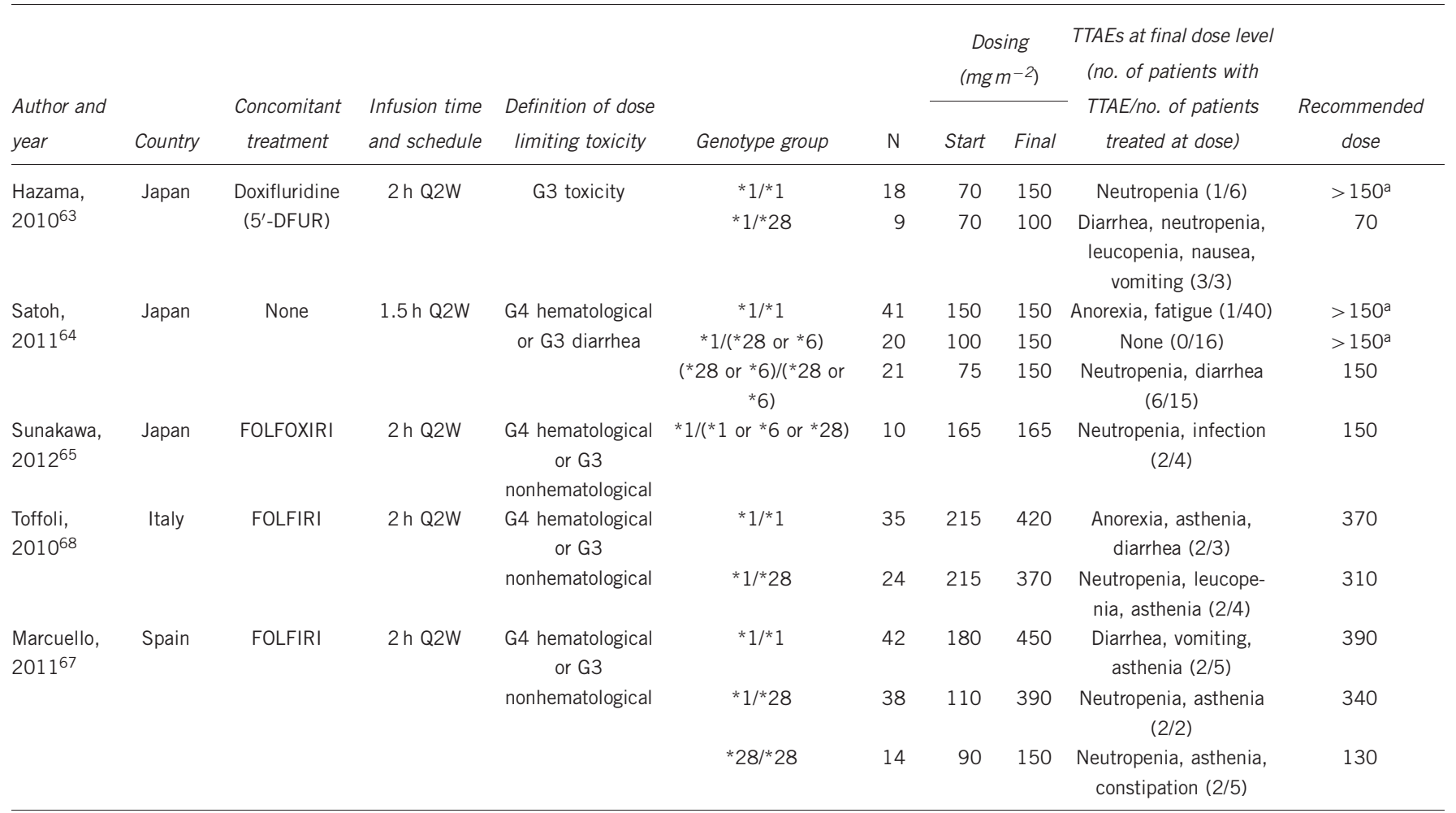

Abbreviations: FOLFIRI, 5-fluorouracil, leucovorin, irinotecan; FOLFOXIRI, 5-fluorouracil, leucovorin, oxaliplatin, irinotecan; Q2W, every 2 weeks; G3, grade 3 or higher; G4, grade 4 or higher; TTAE, treatment terminating adverse event.

aRecommended dose not reached due to dosing restriction limiting dose to $150 \mathrm{mg} \mathrm{m}^{-2}$.

Germline variation could be the underlying cause of any these mechanisms of sensitivity. Regardless of the mechanism, a patient that carried a sensitizing germline variant would not be able to tolerate the dose required for treatment efficacy. Thus, clinical implementation of a germline polymorphism that sensitizes a patient to toxicity is likely to be selection of an alternative treatment agent. In this section, we highlight examples of these three mechanisms of toxicity sensitivity in the context of paclitaxel-induced peripheral neuropathy (PIPN).

Paclitaxel-induced peripheral neuropathy. Peripheral neuropathy is an adverse event commonly associated with treatment from microtubule targeting agents including taxanes and vincas. ${ }^{75}$ The taxanes, including the particularly neuropathic paclitaxel, work by binding to the microtubule $\beta$-tubulin subunit, causing microtubule stability and cellular apoptosis. ${ }^{76}$ The exact mechanism of PIPN is unclear, but may be related to the role of microtubules in neuronal axon guidance and development. ${ }^{77}$ PIPN manifests as parasthesia in the hands and feet, which can progress in severity with continued treatment. As neuropathy is common, debilitating and necessitates treatment discontinuation, prediction of PIPN sensitivity is of great interest to clinicians.

Expression of drug target. $\beta$-Tubulin, the paclitaxel target, exists in multiple isoforms that are differentially expressed throughout the body. $\beta$-Tubulin has garnered some interest as a possible somatic marker of tumor sensitivity, however, somatic variation within the $T U B B$ genes is exceedingly rare and the reported findings are likely due to genotyping errors. ${ }^{78}$ Several $\beta$-tubulin isoforms are expressed in peripheral neurons, the likely site of action causing peripheral neurotoxicity. Leandro-Garcia et al. recently reported an analysis of germline variants within the TUBB2A promoter, which encodes the neuronally expressed $\beta$-tubulin class IIa isotype. They reported an association with a promoter single-nucleotide polymorphism $(-112 \mathrm{~A}>\mathrm{G}$, rs909965), increased gene transcription and protection from PIPN. ${ }^{79}$

Inadequate stimulus response. Pharmacological understanding aside from the mechanism of action may provide hypotheses for investigating cellular sensitivity. As an example, Sucheston et al. looked specifically at the genes encoding two proteins involved in DNA repair, BRCA1 and FANCD2. The investigators selected tag singlenucleotide polymorphisms within each gene to assess as much of the existing genetic variability as possible. Although there were no associations with $B R C A 1$, the authors reported significant associations with neuropathy risk and single-nucleotide polymorphisms and haplotypes within FANCD2. ${ }^{80}$ This same strategy could be applied to other known mechanisms of cellular response to chemotherapeutic agents.

Underlying pathophysiology. Peripheral neuropathy is not only a treatment-related adverse effect; it is a condition that afflicts healthy people that can be hereditarily acquired. Charcot-Marie-Tooth disease is a familial neuropathy condition that has many subtypes, some of which have causative germline variants that have been discovered. Charcot-Marie-Tooth Disease Type $4 \mathrm{H}$ is caused by a variant in the FGD4 gene, which encodes FGD1-related F-actinbinding protein (Frabin). ${ }^{81}$ Patients with other variants in FGD4, or proteins that interact with FGD4, are likely to have subclinical 
neuropathy or extreme sensitivity to chemotherapy-induced neuropathy. A recent genome-wide association study of PIPN discovered an association between an FGD4 polymorphism (rs10771973) and increased neuropathy risk in 855 Caucasian patients $\left(\mathrm{HR}=1.57 \quad(1.13-3.28), \quad P=2.6 \times 10^{-6}\right), \quad$ and then replicated the association in two smaller cohorts of Caucasian $(\mathrm{HR}=1.72 \quad(1.06-2.80), \quad P=0.013) \quad$ and African-American $\left(\mathrm{HR}=1.93(1.13-3.28), P=6.7 \times 10^{-3}\right)$ patients. It is likely that variants in other genes known to be involved in familial neuropathy conditions also influence patient sensitivity to peripheral neuropathy.

\section{CONCLUSION}

The mapping and understanding of the human genome has been a major boon for cancer research. Both the somatic and germline genome can inform treatment decisions. The somatic genome can assist in predicting the tumor's behavior if untreated (prognosis) or treated (efficacy prediction). The germline genome can also influence prognosis and is involved in determining drug exposure and toxicity. The clinical implementation of these pharmacogenetic factors depends on which system they influence. Prognostic factors can be useful in deciding treatment aggressiveness; toxicity and efficacy predictors can be helpful in selecting the appropriate drug for the patient/tumor; and exposure predictors may inform dosage selection. Continued progress in our discovery and translation of these pharmacogenetic factors will someday enable integration of germline and somatic genetic information into individualized treatment decision making for cancer patients.

\section{CONFLICT OF INTEREST}

The authors declare no conflict of interest.

\section{ACKNOWLEDGEMENTS}

Dr Hertz is an American Foundation for Pharmaceutical Education fellow.

Author contributions: D.L.H. and H.L.M. contributed to the

conceptualization of the review. D.L.H. wrote the first draft, and H.L.M. revised it and gave final approval.

1 McLeod, H. L. Cancer pharmacogenomics: early promise, but concerted effort needed. Science 339, 1563-1566 (2013).

2 Gerlinger, M., Rowan, A. J., Horswell, S., Larkin, J., Endesfelder, D., Gronroos, E. et al. Intratumor Heterogeneity and branched evolution revealed by multiregion sequencing. N. Engl. J. Med. 366, 883-892 (2013).

3 Ganem, N. J. \& Pellman, D. Linking abnormal mitosis to the acquisition of DNA damage. J. Cell Biol. 199, 871-881 (2012).

4 Greene, F. L. \& Sobin, L. H. The staging of cancer: a retrospective and prospective appraisal. CA Cancer J. Clin. 58, 180-190 (2008).

5 Sørlie, T., Perou, C. M., Tibshirani, R., Aas, T., Geisler, S., Johnsen, H. et al. Gene expression patterns of breast carcinomas distinguish tumor subclasses with clinical implications. Proc. Natl Acad. Sci. USA 98, 10869-10874 (2001).

6 Nielsen, T. O., Parker, J. S., Leung, S., Voduc, D., Ebbert, M., Vickery, T. et al. A comparison of PAM50 intrinsic subtyping with immunohistochemistry and clinical prognostic factors in tamoxifen-treated estrogen receptor-positive breast cancer. Clin. Cancer Res. 16, 5222-5232 (2010).

7 Goldhirsch, A., Ingle, J. N., Gelber, R. D., Coates, A. S., Thürlimann, B., Senn, H. et al. Thresholds for therapies: highlights of the St Gallen International Expert Consensus on the primary therapy of early breast cancer 2009. Ann. Oncol. 20, 1319-1329 (2009).

8 Paik, S., Shak, S., Tang, G., Kim, C., Baker, J., Cronin, M. et al. A multigene assay to predict recurrence of tamoxifen-treated, node-negative breast cancer. N. Engl. J. Med. 351, 2817-2826 (2004).

9 Albain, K. S., Barlow, W. E., Shak, S., Hortobagyi, G. N., Livingston, R. B., Yeh, I. T. et al. Prognostic and predictive value of the 21-gene recurrence score assay in postmenopausal women with node-positive, oestrogen-receptor-positive breast cancer on chemotherapy: a retrospective analysis of a randomised trial. Lancet Oncol. 11, 55-65 (2010)

10 Sparano, J. A. The TAILORx trial: individualized options for treatment. Comm. Oncol. 83, 494-496 (2006).
11 van't Veer, L. J., Dai, H., van de Vijver, M. J., He, Y. D., Hart, A. A., Mao, M. et al. Gene expression profiling predicts clinical outcome of breast cancer. Nature $\mathbf{4 1 5}, 530-536$ (2002).

12 van de Vijver, M. J., He, Y. D., van't Veer, L. J., Dai, H., Hart, A. A., Voskuil, D. W. et al. A gene-expression signature as a predictor of survival in breast cancer. N. Engl. J. Med. 347, 1999-2009 (2002).

13 Wittner, B. S., Sgroi, D. C., Ryan, P. D., Bruinsma, T. J., Glas, A. M., Male, A. et al. Analysis of the MammaPrint breast cancer assay in a predominantly postmenopausal cohort. Clin. Cancer Res. 14, 2988-2993 (2008).

14 Rutgers, E., Piccart-Gebhart, M. J., Bogaerts, J., Delaloge, S., Veer, L. V., Rubio, I. T. et al. The EORTC 10041/BIG 03-04 MINDACT trial is feasible: results of the pilot phase. Eur. J. Cancer 47, 2742-2749 (2011).

15 Fan, C., Oh, D. S., Wessels, L., Weigelt, B., Nuyten, D. S., Nobel, A. B. et al. Concordance among gene-expression-based predictors for breast cancer. N. Engl. J. Med. 355, 560-569 (2006).

16 Tsimberidou, A. M., Iskander, N. G., Hong, D. S., Wheler, J. J., Falchook, G. S., Fu, S. et al. Personalized medicine in a phase I clinical trials program: the MD Anderson Cancer Center initiative. Clin. Cancer Res. 18, 6373-6383 (2012).

17 Roychowdhury, S., Iyer, M. K., Robinson, D. R., Lonigro, R. J., Wu, Y. M., Cao, X. et al. Personalized oncology through integrative high-throughput sequencing: a pilot study. Sci. Transl. Med. 3, 111 ra121 (2011).

18 Mandrekar, S. J. \& Sargent, D. J. Clinical trial designs for predictive biomarker validation: theoretical considerations and practical challenges. J. Clin. Oncol. 27, 4027-4034 (2009).

19 Inoki, K., Corradetti, M. N. \& Guan, K. L. Dysregulation of the TSC-mTOR pathway in human disease. Nat. Genet. 37, 19-24 (2005).

20 Meikle, L., Pollizzi, K., Egnor, A., Kramvis, I., Lane, H., Sahin, M. et al. Response of a neuronal model of tuberous sclerosis to mammalian target of rapamycin (mTOR) inhibitors: effects on mTORC1 and Akt signaling lead to improved survival and function. J. Neurosci. 28, 5422-5432 (2008).

21 Franz, D. N. Everolimus: an mTOR inhibitor for the treatment of tuberous sclerosis. Expert Rev. Anticancer Ther. 11, 1181-1192 (2011).

22 Iyer, G., Hanrahan, A. J., Milowsky, M. I., Al-Ahmadie, H., Scott, S. N., Janakiraman, M. et al. Genome sequencing identifies a basis for everolimus sensitivity. Science 338, 221 (2012).

23 Davies, H., Bignell, G. R., Cox, C., Stephens, P., Edkins, S., Clegg, S. et al. Mutations of the BRAF gene in human cancer. Nature 417, 949-954 (2002).

24 Tsai, J., Lee, J. T., Wang, W., Zhang, J., Cho, H., Mamo, S. et al. Discovery of a selective inhibitor of oncogenic B-Raf kinase with potent antimelanoma activity. Proc. Natl Acad. Sci. USA 105, 3041-3046 (2008).

25 Flaherty, K. T., Puzanov, I., Kim, K. B., Ribas, A., McArthur, G. A., Sosman, J. A. et al. Inhibition of mutated, activated BRAF in metastatic melanoma. N. Engl. J. Med. 363 , 809-819 (2010).

26 Chapman, P. B., Hauschild, A., Robert, C., Haanen, J. B., Ascierto, P., Larkin, J. et al. Improved survival with vemurafenib in melanoma with BRAF V600E mutation. N. Engl. J. Med. 364, 2507-2516 (2011).

27 Halait, H., Demartin, K., Shah, S., Soviero, S., Langland, R., Cheng, S. et al. Analytical performance of a real-time PCR-based assay for V600 mutations in the BRAF gene, used as the companion diagnostic test for the novel BRAF inhibitor vemurafenib in metastatic melanoma. Diagn Mol. Pathol. 21, 1-8 (2012).

$28 \mathrm{Li}$, F. P. Translational research on hereditary colon, breast, and ovarian cancers. J. Nat Cancer Inst. Monogr. 17, 1-4 (1995).

29 McLeod, H. L. \& Evans, W. E. Pharmacogenomics: unlocking the human genome for better drug therapy. Annu. Rev. Pharmacol. Toxicol. 41, 101-121 (2001).

30 Theodoratou, E., Montazeri, Z., Hawken, S., Allum, G. C., Gong, J., Tait, V. et al. Systematic meta-analyses and field synopsis of genetic association studies in colorectal cancer. J. Natl Cancer Inst. 104, 1433-1457 (2012).

31 Siddiq, A., Couch, F. J., Chen, G. K., Lindstrom, S., Eccles, D., Millikan, R. C. et al. A meta-analysis of genome-wide association studies of breast cancer identifies two novel susceptibility loci at $6 \mathrm{q} 14$ and $20 \mathrm{q} 11$. Hum. Mol. Genet. 21, 5373-5384 (2012).

32 Collins, F. S. \& Barker, A. D. Mapping the cancer genome. Pinpointing the genes involved in cancer will help chart a new course across the complex landscape of human malignancies. Sci. Am. 296, 50-57 (2007).

33 Cancer Genome Atlas Network. Comprehensive molecular characterization of human colon and rectal cancer. Nature 487, 330-337 (2012).

34 Cancer Genome Atlas Research Network. Integrated genomic analyses of ovarian carcinoma. Nature 474, 609-615 (2011).

35 Cancer Genome Atlas Research Network. Comprehensive genomic characterization of squamous cell lung cancers. Nature 489, 519-525 (2012).

36 Cancer Genome Atlas Network. Comprehensive molecular portraits of human breast tumours. Nature 490, 61-70 (2012).

37 Yang, D., Khan, S., Sun, Y., Hess, K., Shmulevich, I., Sood, A. K. et al. Association of BRCA1 and BRCA2 mutations with survival, chemotherapy sensitivity, and gene mutator phenotype in patients with ovarian cancer. JAMA 306, 1557-1565 (2011).

38 Bolton, K. L., Chenevix-Trench, G., Goh, C., Sadetzki, S., Ramus, S. J., Karlan, B. Y. et al. Association between BRCA1 and BRCA2 mutations and survival in women with invasive epithelial ovarian cancer. JAMA 307, 382-390 (2012).

39 Park, Y. G., Zhao, X., Lesueur, F., Lowy, D. R., Lancaster, M., Pharoah, P. et al. Sipa1 is a candidate for underlying the metastasis efficiency modifier locus Mtes1. Nat. Genet. 37, 1055-1062 (2005). 
40 Crawford, N. P., Ziogas, A., Peel, D. J., Hess, J., Anton-Culver, H. \& Hunter, K. W. Germline polymorphisms in SIPA1 are associated with metastasis and other indicators of poor prognosis in breast cancer. Breast Cancer Res. 8, R16 (2006).

41 Pei, R., Xu, Y., Wei, Y., Ouyang, T., Li, J., Wang, T. et al. Association of SIPA1 545 C > T polymorphism with survival in Chinese women with metastatic breast cancer. Front Med. 7, 138-142 (2013).

42 Crawford, N. P., Alsarraj, J., Lukes, L., Walker, R. C., Officewala, J. S., Yang, H. H. et al. Bromodomain 4 activation predicts breast cancer survival. Proc. Natl Acad. Sci. USA 105, 6380-6385 (2008).

43 Crawford, N. P., Qian, X., Ziogas, A., Papageorge, A. G., Boersma, B. J., Walker, R. C. et al. Rrp1b, a new candidate susceptibility gene for breast cancer progression and metastasis. PLoS Genet. 3, e214 (2007).

44 Hsieh, S. M., Look, M. P., Sieuwerts, A. M., Foekens, J. A. \& Hunter, K. W. Distinct inherited metastasis susceptibility exists for different breast cancer subtypes: a prognosis study. Breast Cancer Res. 11, R75 (2009).

45 Shimizu, Y., Hamazaki, Y., Hattori, M., Doi, K., Terada, N., Kobayashi, T. et al. SPA-1 controls the invasion and metastasis of human prostate cancer. Cancer Sci. 102, 828-836 (2011)

46 Lin, J. H. Pharmacokinetic and pharmacodynamic variability: a daunting challenge in drug therapy. Curr. Drug Metab. 8, 109-136 (2007).

47 Felici, A., Verweij, J. \& Sparreboom, A. Dosing strategies for anticancer drugs: the good, the bad and body-surface area. Eur. J. Cancer 38, 1677-1684 (2002).

48 McLeay, S. C., Morrish, G. A., Kirkpatrick, C. M. \& Green, B. The relationship between drug clearance and body size: systematic review and meta-analysis of the literature published from 2000 to 2007. Clin. Pharmacokinet. 51, 319-330 (2012)

49 Krens, S. D., McLeod, H. L. \& Hertz, D. L. Pharmacogenetics, enzyme probes and therapeutic drug monitoring as potential tools for individualizing taxane therapy. Pharmacogenomics 14, 555-574 (2013).

50 Begg, E. J., Helsby, N. A. \& Jensen, B. P. Pharmacogenetics of drug-metabolizing enzymes: the prodrug hypothesis. Pharmacogenomics 13, 83-89 (2012)

51 lyer, L., Hall, D., Das, S., Mortell, M. A., Ramirez, J., Kim, S. et al. Phenotype-genotype correlation of in vitro SN-38 (active metabolite of irinotecan) and bilirubin glucuronidation in human liver tissue with UGT1A1 promoter polymorphism. Clin. Pharmacol. Ther. 65, 576-582 (1999).

52 Ando, Y., Saka, H., Asai, G., Sugiura, S., Shimokata, K. \& Kamataki, T. UGT1A1 genotypes and glucuronidation of SN-38, the active metabolite of irinotecan. Ann. Oncol. 9, 845-847 (1998).

53 Paoluzzi, L., Singh, A. S., Price, D. K., Danesi, R., Mathijssen, R. H., Verweij, J. et al. Influence of genetic variants in UGT1A1 and UGT1A9 on the in vivo glucuronidation of SN-38. J. Clin. Pharmacol. 44, 854-860 (2004).

54 Stewart, C. F., Panetta, J. C., O'Shaughnessy, M. A., Throm, S. L., Fraga, C. H., Owens, T. et al. UGT1A1 promoter genotype correlates with $\mathrm{SN}-38$ pharmacokinetics, but not severe toxicity in patients receiving low-dose irinotecan. J. Clin. Oncol. 25, 2594-2600 (2007)

55 Toffoli, G., Cecchin, E., Corona, G., Russo, A., Buonadonna, A., D'Andrea, M. et al. The role of UGT1A1*28 polymorphism in the pharmacodynamics and pharmacokinetics of irinotecan in patients with metastatic colorectal cancer. J. Clin. Oncol. 24 3061-3068 (2006)

56 Saito, Y., Sai, K., Maekawa, K., Kaniwa, N., Shirao, K., Hamaguchi, T. et al. Close association of UGT1A9 IVS1 + 399C $>$ T with UGT1A1*28, *6, or *60 haplotype and its apparent influence on 7-ethyl-10-hydroxycamptothecin (SN-38) glucuronidation in Japanese. Drug Metab. Dispos. 37, 272-276 (2009).

57 Minami, H., Sai, K., Saeki, M., Saito, Y., Ozawa, S., Suzuki, K. et al. Irinotecan pharmacokinetics/pharmacodynamics and UGT1A genetic polymorphisms in Japanese: roles of UGT1A1*6 and *28. Pharmacogenet. Genomics 17, 497-504 (2007).

58 Yamamoto, N., Takahashi, T., Kunikane, H., Masuda, N., Eguchi, K., Shibuya, M. et al. Phase I/II pharmacokinetic and pharmacogenomic study of UGT1A1 polymorphism in elderly patients with advanced non-small cell lung cancer treated with irinotecan. Clin. Pharmacol. Ther. 85, 149-154 (2009).

59 Sai, K., Saeki, M., Saito, Y., Ozawa, S., Katori, N., Jinno, H. et al. UGT1A1 haplotypes associated with reduced glucuronidation and increased serum bilirubin in irinotecanadministered Japanese patients with cancer. Clin. Pharmacol. Ther. 75, 501-515 (2004).

60 Ramchandani, R. P., Wang, Y., Booth, B. P., Ibrahim, A., Johnson, J. R., Rahman, A. et al. The role of $\mathrm{SN}-38$ exposure, UGT1A1*28 polymorphism, and baseline bilirubin level in predicting severe irinotecan toxicity. J. Clin. Pharmacol. 47, 78-86 (2007).
61 Evaluation of Genomic Applications in Practice and Prevention (EGAPP) Working Group. Recommendations from the EGAPP Working Group: can UGT1A1 genotyping reduce morbidity and mortality in patients with metastatic colorectal cancer treated with irinotecan? Genet. Med. 11, 15-20 (2009).

62 Di Paolo, A., Bocci, G., Polillo, M., Del Re, M., Di Desidero, T., Lastella, M. et al. Pharmacokinetic and pharmacogenetic predictive markers of irinotecan activity and toxicity. Curr. Drug Metab. 12, 932-943 (2011).

63 Hazama, S., Nagashima, A., Kondo, H., Yoshida, S., Shimizu, R., Araki, A. et al. Phase I study of irinotecan and doxifluridine for metastatic colorectal cancer focusing on the UGT1A1*28 polymorphism. Cancer Sci. 101, 722-727 (2010).

64 Satoh, T., Ura, T., Yamada, Y., Yamazaki, K., Tsujinaka, T., Munakata, M. et al. Genotype-directed, dose-finding study of irinotecan in cancer patients with UGT1A1*28 and/or UGT1A1*6 polymorphisms. Cancer Sci. 102, 1868-1873 (2011).

65 Sunakawa, Y., Fujita, K., Ichikawa, W., Ishida, H., Yamashita, K., Araki, K. et al. A phase I study of infusional 5-fluorouracil, leucovorin, oxaliplatin and irinotecan in Japanese patients with advanced colorectal cancer who harbor UGT1A1 $1 /{ }^{*} 1,{ }^{*} 1 / * 6$ or *1/*28. Oncology 82, 242-248 (2012).

66 Okuyama, Y., Hazama, S., Nozawa, H., Kobayashi, M., Takahashi, K., Fujikawa, K. et al. Prospective phase II study of FOLFIRI for mCRC in Japan, including the analysis of UGT1A1 28/6 polymorphisms. Jpn. J. Clin. Oncol. 41, 477-482 (2011).

67 Marcuello, E., Paez, D., Pare, L., Salazar, J., Sebio, A., del Rio, E. et al. A genotype directed phase I-IV dose-finding study of irinotecan in combination with fluorouracil/ leucovorin as first-line treatment in advanced colorectal cancer. Br. J. Cancer 105 53-57 (2011).

68 Toffoli, G., Cecchin, E., Gasparini, G., D’Andrea, M., Azzarello, G., Basso, U. et al. Genotype-driven phase I study of irinotecan administered in combination with fluorouracil/leucovorin in patients with metastatic colorectal cancer. J. Clin. Oncol. 28, 866-871 (2010).

69 Freyer, G., Duret, A., Milano, G., Chatelut, E., Rebischung, C., Delord, J. P. et al. Pharmacogenetic tailoring of irinotecan-based first-line chemotherapy in metastatic colorectal cancer: results of a pilot study. Anticancer Res. 31, 359-366 (2011).

70 de Jong, F. A., Mathijssen, R. H., Xie, R., Verweij, J. \& Sparreboom, A. Flat-fixed dosing of irinotecan: influence on pharmacokinetic and pharmacodynamic variability. Clin. Cancer Res. 10 (Part 1), 4068-4071 (2004).

71 Mathijssen, R. H., Marsh, S., Karlsson, M. O., Xie, R., Baker, S. D., Verweij, J. et al. Irinotecan pathway genotype analysis to predict pharmacokinetics. Clin. Cancer Res. 9 , 3246-3253 (2003)

72 Innocenti, F., Kroetz, D. L., Schuetz, E., Dolan, M. E., Ramirez, J., Relling, M. et al. Comprehensive pharmacogenetic analysis of irinotecan neutropenia and pharmacokinetics. J. Clin. Oncol. 27, 2604-2614 (2009).

73 Sai, K., Saito, Y., Tatewaki, N., Hosokawa, M., Kaniwa, N., Nishimaki-Mogami, T. et al. Association of carboxylesterase $1 \mathrm{~A}$ genotypes with irinotecan pharmacokinetics in Japanese cancer patients. Br. J. Clin. Pharmacol. 70, 222-233 (2010).

74 Sai, K., Saito, Y., Fukushima-Uesaka, H., Kurose, K., Kaniwa, N., Kamatani, N. et al. Impact of CYP3A4 haplotypes on irinotecan pharmacokinetics in Japanese cancer patients. Cancer Chemother. Pharmacol. 62, 529-537 (2008)

75 Lee, J. J. \& Swain, S. M. Peripheral neuropathy Induced by microtubule-stabilizing agents. J. Clin. Oncol. 24, 1633-1642 (2006).

76 Wilson, L. \& Jordan, M. A. Microtubule dynamics: taking aim at a moving target. Chem. Biol. 2, 569-573 (1995).

77 Bamburg, J. R., Bray, D. \& Chapman, K. Assembly of microtubules at the tip of growing axons. Nature 321, 788-790 (1986).

78 Berrieman, H. K., Lind, M. J. \& Cawkwell, L. Do $\beta$-tubulin mutations have a role in resistance to chemotherapy? Lancet Oncol. 5, 158-164 (2004).

79 Leandro-Garcia, L. J., Leskela, S., Jara Sanchez, C., Green, H., Avall Lundqvist, E., Wheeler, H. E. et al. Regulatory polymorphisms in beta-tubulin Ila are associated with paclitaxel-induced peripheral neuropathy. Clin. Cancer Res. 18, 4441-4448 (2012).

80 Sucheston, L., Zhao, H., Yao, S., Zirpoli, G., Liu, S., Barlow, W. et al. Genetic predictors of taxane-induced neurotoxicity in a SWOG phase III intergroup adjuvant breast cancer treatment trial (S0221). Breast Cancer Res. Treat. 130, 993-1002 (2011).

81 Delague, V., Jacquier, A., Hamadouche, T., Poitelon, Y., Baudot, C., Boccaccio, I. et al. Mutations in FGD4 encoding the Rho GDP/GTP exchange factor FRABIN cause autosomal recessive Charcot-Marie-Tooth type 4H. Am. J. Hum. Genet. 81, 1-16 (2007). 